\title{
Super Rogue Waves: Observation of a Higher-Order Breather in Water Waves
}

\author{
A. Chabchoub, ${ }^{1, *}$ N. Hoffmann, ${ }^{1}$ M. Onorato, ${ }^{2,3}$ and N. Akhmediev ${ }^{4}$ \\ ${ }^{1}$ Mechanics and Ocean Engineering, Hamburg University of Technology Eißendorfer Straße 42, 21073 Hamburg, Germany \\ ${ }^{2}$ Dipartimento di Fisica, Università degli Studi di Torino, Torino 10125, Italy \\ ${ }^{3}$ Istituto Nazionale di Fisica Nucleare, INFN, Sezione di Torino, Torino 10125, Italy \\ ${ }^{4}$ Optical Sciences Group, Research School of Physics and Engineering, The Australian National University, \\ Canberra ACT 0200, Australia
}

(Received 17 January 2012; revised manuscript received 28 February 2012; published 29 March 2012)

\begin{abstract}
Super rogue waves with an amplitude of up to 5 times the background value are observed in a waterwave tank for the first time. Nonlinear focusing of the local wave amplitude occurs according to the higher-order breather solution of the nonlinear wave equation. The present result shows that rogue waves can also develop from very calm and apparently safe sea states. We expect the result to have a significant impact on studies of extreme ocean waves and to initiate related studies in other disciplines concerned with waves in nonlinear dispersive media, such as optics, plasma physics, and superfluidity.
\end{abstract}

DOI: 10.1103/PhysRevX.2.011015

Subject Areas: Fluid Dynamics, Geophysics, Nonlinear Dynamics

\section{INTRODUCTION}

The nature of rogue waves (RWs) has been discussed in the literature for more than a decade [1-4]. A few major approaches have been suggested to explain the high-impact power of these "monsters of the deep" [5]. Theories vary depending on the conditions where these waves appear $[6,7]$. One remarkable feature of rogue waves is that they appear visibly from nowhere and disappear without a trace. Nonlinear dynamics is one of the approaches that has been successful in predicting the basic features of rogue waves [8-11]. One of the prototypes suggested to model rogue waves is the so-called Peregrine soliton $[12,13]$. The reason is that such a solution describes the growing evolution of a small, localized perturbation of a plane wave with the subsequent peak amplification of 3 above the plane wave. The large-amplitude peak appears just once in evolution (it is doubly localized rather than periodic in space and time). Despite decades of debate $[5,6,8,14]$, only very recently was the fundamental Peregrine breather soliton observed experimentally in fiber optics [15]. Soon after, it was observed in a water-wave tank [16] and a few months later it was observed in multicomponent plasma [17]. These observations proved that the nonlinear approach is fruitful in a description of rogue waves. The nonlinear theory also predicts that, in addition to the unique fundamental Peregrine soliton, there is an infinite hierarchy of higherorder breather solutions with a progressively increasing amplitude $[18,19]$ that are also localized both in space and time. The study of these solutions is crucial in explaining the even higher amplitude waves that can be observed

\footnotetext{
*amin.chabchoub@tuhh.de
}

Published by the American Physical Society under the terms of the Creative Commons Attribution 3.0 License. Further distribution of this work must maintain attribution to the author(s) and the published article's title, journal citation, and DOI. in deep-water conditions. Our present experimental study shows that higher-order RWs do exist and can be successfully generated physically in a water-wave experiment. These observations may have far reaching consequences in our efforts to understand the waves that are, by far, still being characterized as "mysterious."

\section{MATHEMATICS AND EXPERIMENT}

The nonlinear Schrödinger equation (NLS) is one of the basic approaches used to describe the nonlinear wave evolution in various media [20,21]. In particular, this equation describes gravity waves in deep water [22-24]:

$$
i\left(\frac{\partial A}{\partial t}+c_{g} \frac{\partial A}{\partial x}\right)-\frac{\omega_{0}}{8 k_{0}^{2}} \frac{\partial^{2} A}{\partial x^{2}}-\frac{\omega_{0} k_{0}^{2}}{2}|A|^{2} A=0,
$$

where $t$ and $x$ are the time and longitudinal coordinates, while $k_{0}$ and $\omega_{0}=\omega\left(k_{0}\right)$ denote the wave number and the frequency of the carrier wave, respectively, which are connected through the dispersion relation of linear deep-water wave theory, $\omega_{0}=\sqrt{g k_{0}}$, where $g$ is the gravitational acceleration. The group velocity here is $c_{g}:=\frac{d \omega}{d k}=\frac{\omega_{0}}{2 k_{0}}$.

The surface elevation $\eta(x, t)$ is related to the NLS variable $A(x, t)$ to second order in steepness as follows:

$$
\begin{aligned}
\eta(x, t)= & \operatorname{Re}\left\{A(x, t) \exp \left[i\left(k_{0} x-\omega_{0} t\right)\right]\right\} \\
& +\operatorname{Re}\left\{\frac{1}{2} k_{0} A^{2}(x, t) \exp \left[2 i\left(k_{0} x-\omega_{0} t\right)\right]\right\} .
\end{aligned}
$$

A dimensionless form of the NLS,

$$
i \psi_{T}+\psi_{X X}+2|\psi|^{2} \psi=0
$$

is obtained from (1) using the rescaled variables:

$$
T=-\frac{\omega_{0}}{8} t, \quad X=\left(x-c_{g} t\right) k_{0}=x k_{0}-\frac{\omega_{0}}{2} t, \quad \psi=\sqrt{2} k_{0} A .
$$


Here, $X$ is the coordinate in the frame moving with the wave-group velocity and $T$ is the scaled time.

The NLS equation has a hierarchy of localized breathing solutions with a single maximum [18,25], which can model rogue waves in deep water. For a given carrier amplitude proportional to $\psi_{0}$, they are given in terms of polynomials $G(X, T), H(X, T)$, and $D(X, T)$ :

$$
\psi(X, T)=\psi_{0}\left(1-\frac{G+i H}{D}\right) \exp \left(2 i\left|\psi_{0}\right|^{2} T\right),
$$

where $\psi_{0}$ is a background-amplitude-free parameter. In the simplest case, the first order solution is defined by $G=4$, $H=16\left|\psi_{0}\right|^{2} T, \quad$ and $D=1+4\left|\psi_{0}\right|^{2} X^{2}+16\left|\psi_{0}\right|^{4} T^{2}$. Then (5) is the well-known Peregrine soliton [12,13]. It is a solution that is the limiting case of the space-periodic Akhmediev breather [26] and the time-periodic Kuznetsov-Ma breather $[27,28]$ when the period tends to infinity. This solution is localized in space and time and the maximal amplitude amplification obtained at $X=0$ and $T=0$ is 3 times the background amplitude. The higherorder rational solution, localized in both space and time, was presented in $[18,25]$ and the polynomials for this solution are

$$
G=\left(\left|\psi_{0}\right|^{2} X^{2}+4\left|\psi_{0}\right|^{4} T^{2}+\frac{3}{4}\right)\left(\left|\psi_{0}\right|^{2} X^{2}+20\left|\psi_{0}\right|^{4} T^{2}+\frac{3}{4}\right)-\frac{3}{4},
$$

$$
\begin{gathered}
H=2\left|\psi_{0}\right|^{2} T\left(4\left|\psi_{0}\right|^{4} T^{2}-3\left|\psi_{0}\right|^{2} X^{2}\right) \\
\quad+2\left|\psi_{0}\right|^{2} T\left[2\left(\left|\psi_{0}\right|^{2} X^{2}+4\left|\psi_{0}\right|^{4} T^{2}\right)^{2}-\frac{15}{8}\right] \\
\begin{aligned}
D= & \frac{1}{3}\left(\left|\psi_{0}\right|^{2} X^{2}+4\left|\psi_{0}\right|^{4} T^{2}\right)^{3}+\frac{1}{4}\left(\left|\psi_{0}\right|^{2} X^{2}-12\left|\psi_{0}\right|^{4} T^{2}\right)^{2} \\
& +\frac{3}{64}\left(12\left|\psi_{0}\right|^{2} X^{2}+176\left|\psi_{0}\right|^{4} T^{2}+1\right) .
\end{aligned}
\end{gathered}
$$

A remarkable property of this solution is that it boosts the peak amplitude of the carrier wave at $X=0$ and $T=0$ by

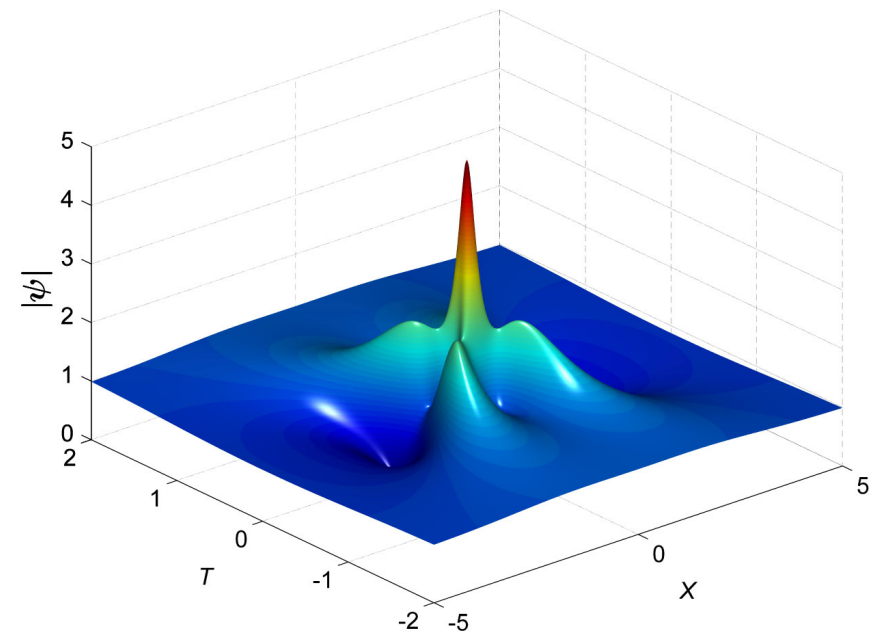

FIG. 1. Higher-order Akhmediev-Peregrine rational solution of the NLS given by Eqs. (5)-(8) with the following background: $\psi_{0}=1$. The maximal amplification of five above the background wave can be observed at $X=0$ and $T=0$. a factor of 5 (see Fig. 1). As such, it describes rogue waves that are higher than the standard Peregrine soliton, the latter being characterized by an amplification factor of 3 . In terms of water waves, this is a significantly higher elevation.

In this work, we present the first observation of the higherorder rogue wave, which is obtained in the water-wave experiments. The tank in which the experiments were performed is $15 \mathrm{~m} \times 1.6 \mathrm{~m} \times 1.5 \mathrm{~m}$. Figure 2 shows a photo and a schematic illustration of our equipment. A single-flap wave-producing paddle activated by a hydraulic cylinder is located at the far end of the tank. The assumption that the flap displacement is proportional to the generated surface height has been checked by measurements and validated by the subsequent results. To avoid wave reflections, an absorbing beach is installed at the opposite end. All experiments are conducted in deep-water conditions, the ratio of the water depth of $1 \mathrm{~m}$ to the wavelength is much larger than unity. The water-surface elevation at any given point is measured by a capacitance wave gauge with a sensitivity of $1.06 \mathrm{~V} / \mathrm{cm}$ and a sampling frequency of $0.5 \mathrm{kHz}$.

In order to excite the super rogue wave in the tank, one has to fix the initial amplitude $a_{0}$ and the wave number of the carrier wave $k_{0}$. Those two parameters define the steepness. Then, using the relation, $\psi_{0}=a_{0} \sqrt{2} k_{0}$, and inverting the scaling in Eq. (4), the analytical solution is written in dimensional form and shifted in space in order to observe the formation of the rogue wave at any desired location in the wave tank. This automatically furnishes the solution at
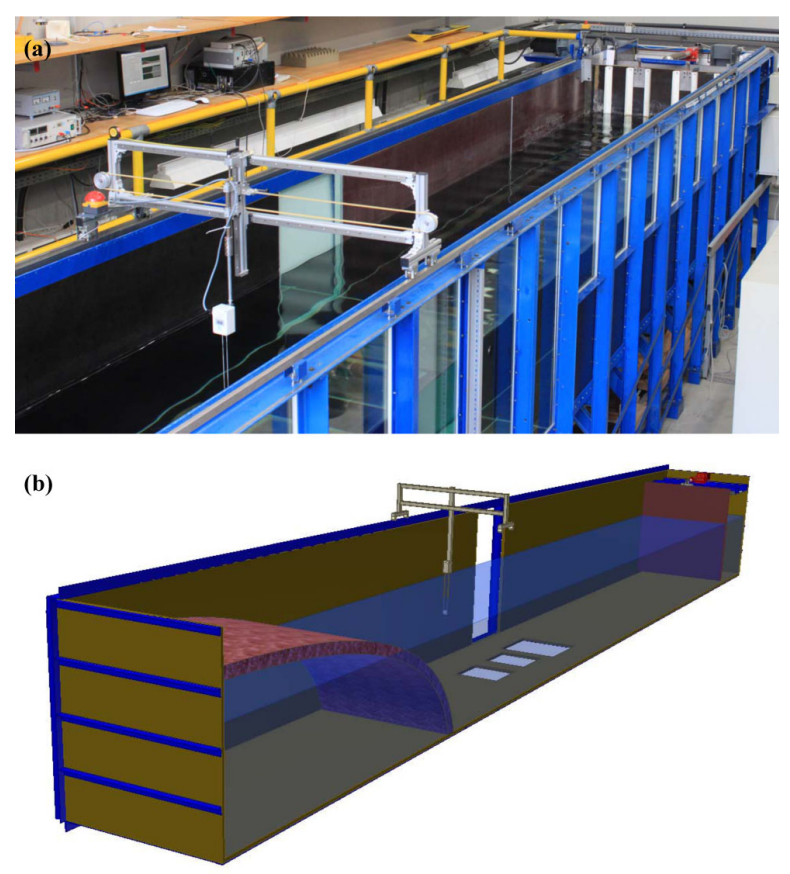

FIG. 2. (a) Photo of the water-wave tank with the surfaceelevation gauge. Waves are generated with the paddle located at the far end of the tank. (b) Schematic illustration of the facility including the paddle, the gauge, and the beach. 
the wave maker and consequently the signal that drives the paddle.

\section{OBSERVATIONS OF THE HIGHER-ORDER ROGUE WAVE}

Our experiments are conducted for a carrier amplitude of $a_{0}=0.001 \mathrm{~m}$ and a steepness of $\varepsilon=a_{0} k_{0}=0.03$; the corresponding wavelength is $20 \mathrm{~cm}$. Close to the wave maker, at a distance of $1 \mathrm{~m}$, the regular background wave is locally perturbed in the middle of the wave train. The parameters of our wave tank, in particular, its limited length, unfortunately, do not allow us to directly observe the full evolution of the higher-order breather from a very small amplitude to its maximum in a single run. As the growth of the amplitude is algebraic rather than exponential, even the use of longer wave tanks remain problematic in this sense. To overcome this difficulty, we split the experiment into several stages. Namely, starting the wave generation repetitively with different boundary conditions given from theory, we measured the wave profile at the other end of the tank. Each time, we check that the final profile follows the theoretical prediction. Deviations from theory found by the direct superposition of theoretical and experimental curves are minimal and mainly feature in the left-right asymmetry of the profiles.

We repeat this process 7 times, thus multiplying the propagation length of 9 meters 8 times. This way we reach the propagation distance of $72 \mathrm{~m}$, which corresponds to the point of maximum amplitude. The left panel in Fig. 3 shows the profiles measured at the end of each propagation segment. The right-hand side panel in Fig. 3 shows the
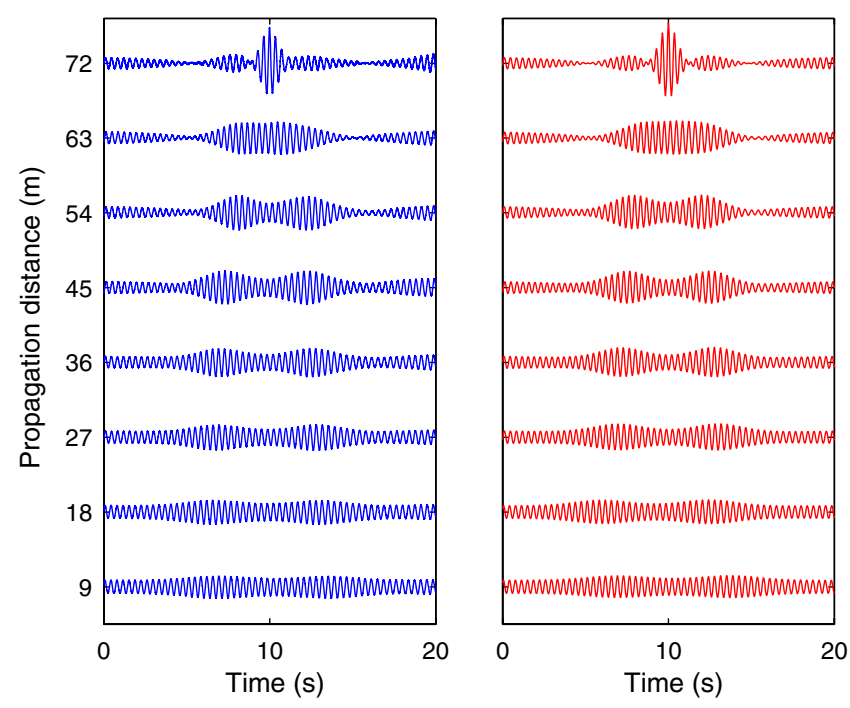

FIG. 3. Wave-profile evolution in the experiment with split propagation. The left panel shows experimental profiles at the end of each segment, while the right panel shows theoretical ones. Up to a distance of $63 \mathrm{~m}$, the curves are almost identical. The maximum amplitude of the higher-order breather is reached at the end of the total propagation distance of $72 \mathrm{~m}$.

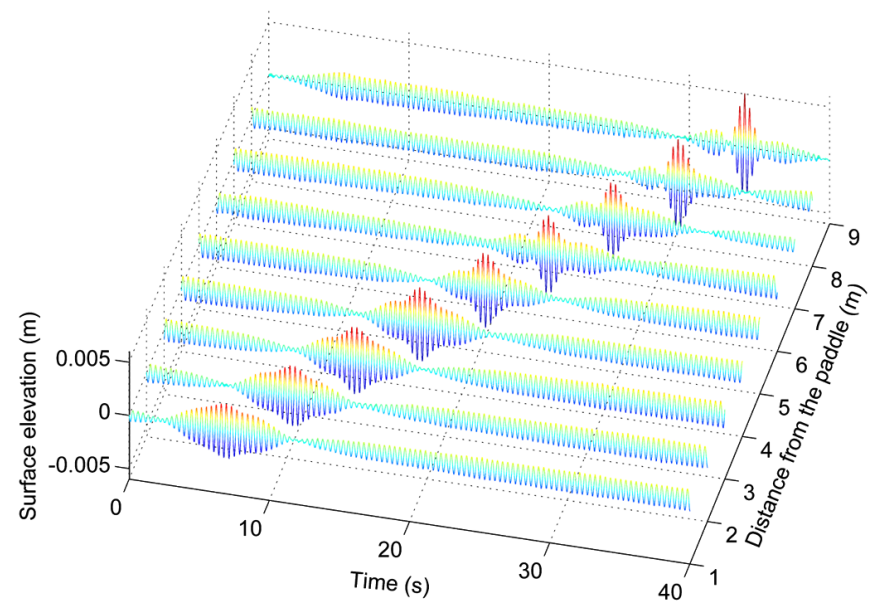

FIG. 4. Final stage of the evolution of the temporal profile of the water-surface elevation along the wave tank demonstrating the super-RW formation at $X=9$ (in total $72 \mathrm{~m}$ ).

corresponding theoretical curves. The comparison between the measured wave profiles and the theoretical curves shows very good agreement and basically justifies our approach.

A more detailed set of profiles of the final stage of evolution in this experiment, effectively from 63 to $72 \mathrm{~m}$ is shown in Fig. 4. As it was prearranged with the initial conditions, the maximal amplitude amplification is reached at the end of the total distance of $x=72 \mathrm{~m}$ (or $9 \mathrm{~m}$ from the beginning of the last span).

The evolution of the surface elevation spectra at the last stage of the experiment is shown in Fig. 5. Only two curves at the beginning and at the end of the segment are shown for clarity. The very fact of a change of spectra in propagation shows clearly that the evolution process is nonlinear. The shape of the highest part of the spectrum (around the

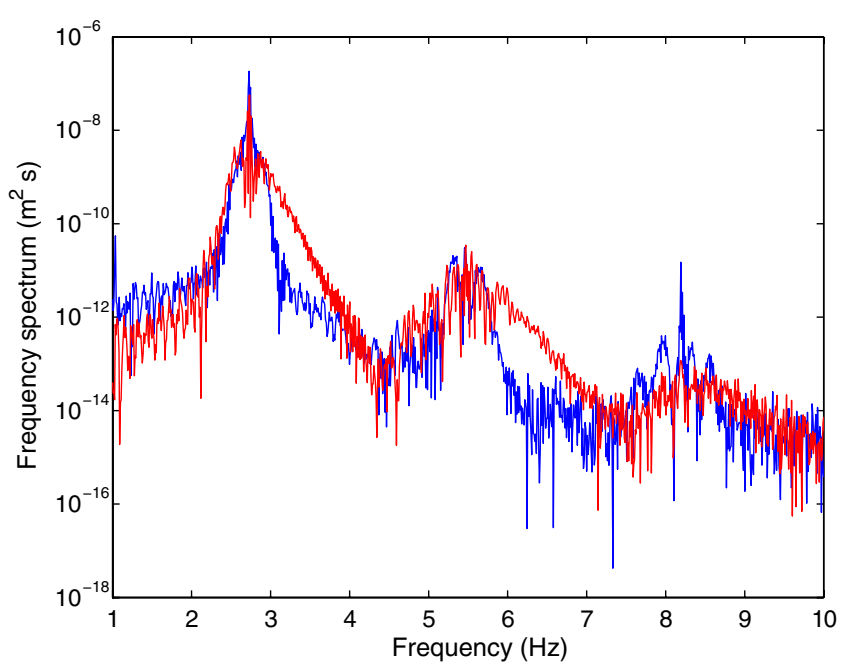

FIG. 5. Spectral profiles of the surface elevation at the last stage of the experiment shown in Fig. 4 at $x=1 \mathrm{~m}$ (red curve) and at $x=9 \mathrm{~m}$ (blue curve). 
frequency of $2.74 \mathrm{~Hz}$ ), which is triangular, agrees well with spectra expected for the second-order rogue wave. The latter have been presented in Fig. 2 of [29]. Two additional peaks of the spectra (around $5.48 \mathrm{~Hz}$ and $8.22 \mathrm{~Hz}$ ) that also have a triangular shape are the second and the third harmonics of the main part of the spectrum. They represent the higher harmonics bound to the primary Stokes wave. Importantly, their amplitudes are 2 orders of magnitude smaller than for the wave at the fundamental frequency.

Figure 6 shows the comparison of the theoretical prediction at the position of maximal amplitude with the measured surface elevation. The free surface elevation has been calculated using Eq. (2). Indeed, the experimental value of the amplification is very close to the expected factor of 5. The destructive impact of such a high wave in a scale of our wave tank is exemplified in Video 1.
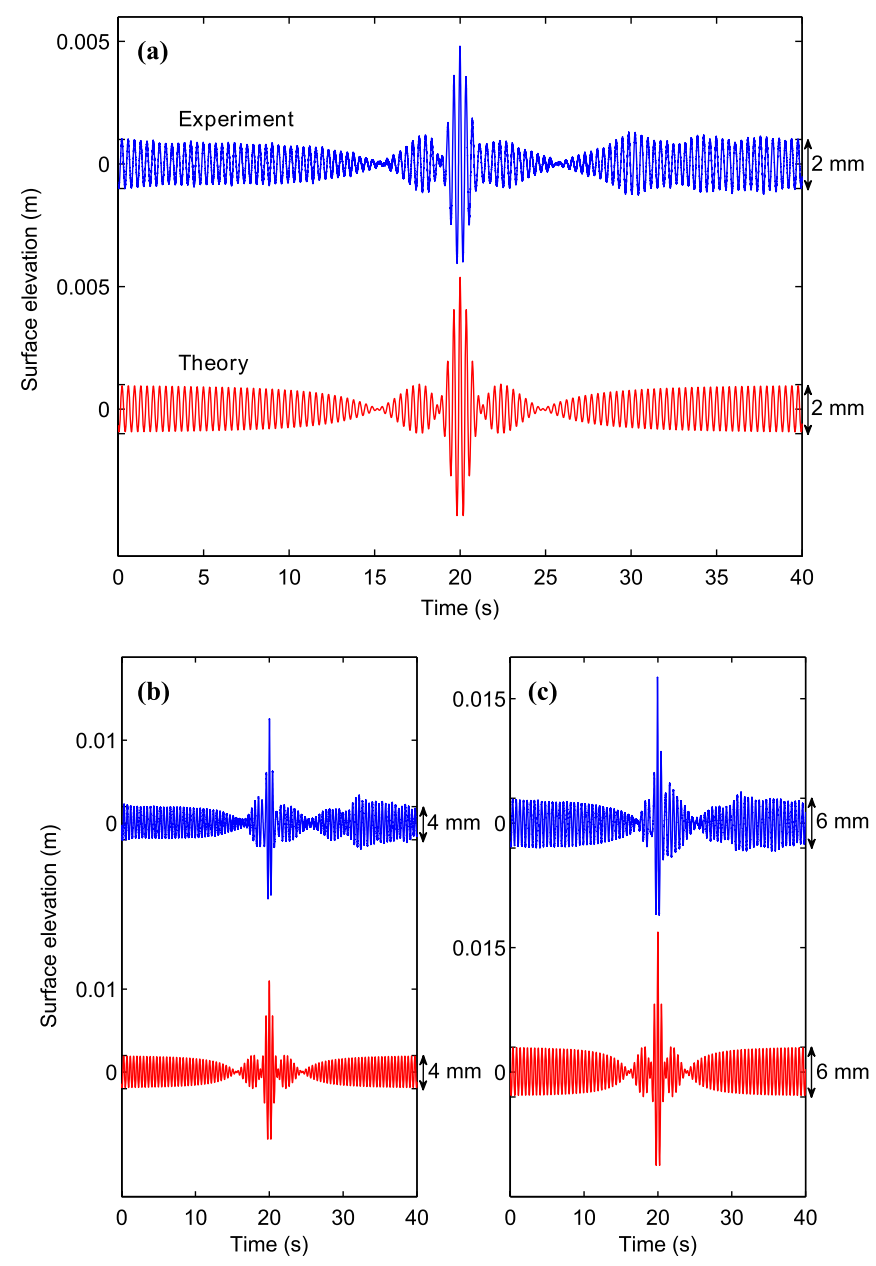

FIG. 6. Three clear observations of the higher-order RWs. Top (blue) curves: Measured wave profile at the expected position of the maximal carrier amplification, (a) for the background amplitude $a_{0}=0.001 \mathrm{~m}$ and the steepness $\varepsilon=0.03$, (b) for $a_{0}=$ $0.002 \mathrm{~m}$ and $\varepsilon=0.04$, and (c) for $a_{0}=0.003 \mathrm{~m}$ and $\varepsilon=0.05$. For comparison, the bottom (red) curves correspond to the theoretically predicted temporal profile at the same position.

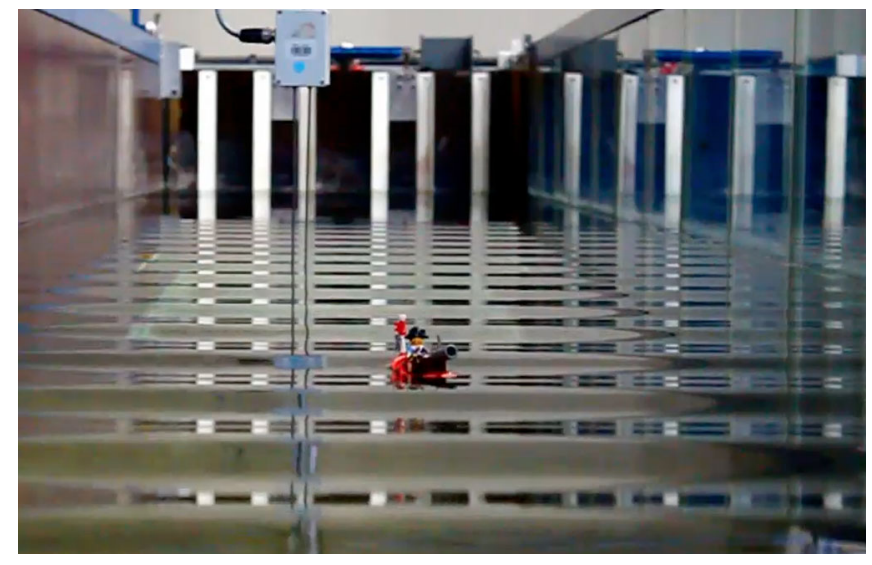

VIDEO 1. Experimental demonstration of the generation and destructive impact of a super rogue wave in a wave tank. The experiment was conducted for a carrier amplitude of $0.003 \mathrm{~m}$ and a steepness of 0.05 . A $10 \mathrm{~cm}$ long toy boat is placed $9 \mathrm{~m}$ from the wave maker, where the super rogue wave reaches its maximal amplification of a factor of 5. First, the boat floats on a regular wave train. Then, the high-order wave packet generated by the wave maker's paddle evolves to a super rogue wave and the toy boat capsizes after being hit by the extreme wave.

The number of modulated waves, as well as the overall wave shape at the left-hand side of the peak amplitude, is almost identical to those obtained from the theoretical higher-order rogue wave solution. Clearly, NLS provides a good basic model for the nonlinear wave propagation. Deviations at the right-hand side of the curve can be attributed to higher-order effects that cannot be captured within the NLS approximation. As expected, the agreement becomes worse when nonlinearity, given by the steepness of the initial condition, becomes higher. Figures 6(b) and 6(c) show the results for $a_{0}=0.002 \mathrm{~m}$, $\varepsilon=0.04$ and $a_{0}=0.003 \mathrm{~m}, \varepsilon=0.05$, respectively. An experimentally observed asymmetry between the waves before and after the rogue wave can be explained in terms of higher-order NLS equations with odd terms such as Dysthe [30] or similar [31] equations that account for the formation of asymmetric wave packets. Moreover, for steepness values of 0.06 and above, we can clearly observe the wave breaking at the top of the rogue wave. Despite these deviations, in all three cases presented above, the wave amplitude at the maximum of the excitation considerably exceeds the amplification of 2.2 above the significant wave height, which is the common definition of a rogue wave.

\section{SUMMARY}

To conclude, the existence of the higher-order rogue wave, a localized wave event with an amplitude of 5 times the background wave, has been proven experimentally in the case of water-surface gravity waves. When the steepness is small (less than 0.03), the agreement between the 
NLS theory and the observation is close to excellent. For larger steepness values (less than 0.06), the observed waves can still be related well to the theoretical breather solution. The large amplification of the peak amplitude above the background wave height by a factor of 5 suggests the existence of a new class of waves to be called "super rogue waves." The second-order RW solution observed in the present study can be considered the first representative of this class. Our results show that, even in a sea state characterized by a very small steepness (of the order of 0.03 ), rogue waves can naturally develop due to the nonlinear dynamics of the surface elevation. This is an extraordinary fact that could explain some mysterious observations of RWs in calm sea states (one example is shown on the cover of [6]). Of course, in real oceans, we have to take into account the second-horizontal dimension and the problem needs more careful analysis. Nevertheless, our observation of the second-order breather is important from the general scientific viewpoint. In purely 1D systems, e.g., in fiber optics, higher-order rogue waves are also possible and could be observed without significant complications. Thus, our present work may stimulate research in other fields concerned with nonlinear dispersive media, e.g., in optics, plasma physics, and superfluidity.

\section{ACKNOWLEDGMENTS}

N.H. and N.A. acknowledge the support of the Volkswagen Stiftung. N. A. acknowledges partial support of the Australian Research Council (Discovery Project No. DP110102068). N.A. acknowledges support from the Alexander von Humboldt Foundation. M. O. was supported by ONR Grant No. N000141010991 and by the European Union under the project EXTREME SEAS (SCP8-GA-2009-234175).

[1] L. Draper, Freak Ocean Waves, Mar. Obs. 35, 193 (1965).

[2] R.G. Dean, Water Wave Kinematics, edited by A. Torum and O.T. Gudmestad (Kluwer, Dordrecht, 1990).

[3] P. Müller, C. Garrett, and A. Osborne, Rogue Waves-The Fourteens 'Aha Huloko'a Hawaiian Winter Workshop, Oceanography 18, 66 (2005).

[4] S. Perkins, Dashing Rogues: Freak Ocean Waves Pose Threat to Ships, Deep-Sea Oil Platforms, Science News (Washington DC) 170, 328 (2006).

[5] C. Kharif, E. Pelinovsky, and A. Slunyaev, Rogue Waves in the Ocean (Springer, Heidelberg, 2009).

[6] N. Akhmediev and E. Pelinovsky, Rogue Waves-Towards a Unifying Concept?: Discussions and Debates, Eur. Phys. J. Special Topics 185, 5 (2010).

[7] M. Erkintalo, G. Genty, and J. M. Dudley, Rogue-WaveLike Characteristics in Femtosecond Supercontinuum Generation, Opt. Lett. 34, 2468 (2009).
[8] K. B. Dysthe and K. Trulsen, Note on Breather Type Solutions of the NLS as Models for Freak-Waves, Phys. Scr. T82, 48 (1999).

[9] A. Osborne, M. Onorato, and M. Serio, The Nonlinear Dynamics of Rogue Waves and Holes in Deep-Water Gravity Wave Train, Phys. Lett. A 275, 386 (2000).

[10] M. Onorato, A. Osborne, M. Serio, and S. Bertone, Freak Wave in Random Oceanic Sea States, Phys. Rev. Lett. 86, 5831 (2001).

[11] C. Garrett and J. Gemmrich, Rogue Waves, Phys. Today 62, 62 (2009).

[12] D.H. Peregrine, Water waves, Nonlinear Schrödinger Equations and their Solutions, J. Aust. Math. Soc. Series B, Appl. Math. 25, 16 (1983).

[13] V. I. Shrira and V. V. Geogjaev, What Makes the Peregrine Soliton So Special as a Prototype of Freak Waves? J. Eng. Math. 67, 11 (2009).

[14] A. Osborne, Nonlinear Ocean Waves and the Inverse Scattering Transform (Elsevier, Amsterdam, 2010).

[15] B. Kibler, J. Fatome, C. Finot, G. Millot, F. Dias, G. Genty, N. Akhmediev, and J. M. Dudley, The Peregrine Soliton in Nonlinear Fibre Optics, Nature Phys. 6, 790 (2010).

[16] A. Chabchoub, N. P. Hoffmann, and N. Akhmediev, Rogue Wave Observation in a Water Wave Tank, Phys. Rev. Lett. 106, 204502 (2011).

[17] H. Bailung, S. K. Sharma, and Y. Nakamura, Observation of Peregrine Solitons in a Multicomponent Plasma with Negative Ions, Phys. Rev. Lett. 107, 255005 (2011).

[18] N. Akhmediev, A. Ankiewicz, and M. Taki, Waves that Appear from Nowhere and Disappear Without a Trace, Phys. Lett. A 373, 675 (2009).

[19] N. Akhmediev, A. Ankiewicz, and J. M. Soto-Crespo, Rogue Waves and Rational Solutions of the Nonlinear Schrödinger Equation, Phys. Rev. E 80, 026601 (2009).

[20] R. Y. Chiao, E. Garmire, and C. H. Towns, Self-Trapping of Optical Beams, Phys. Rev. Lett. 13, 479 (1964).

[21] D. J. Benney and A.C. Newell, The Propagation of Nonlinear Wave Envelopes, J. Math. Phys. (N.Y.) 46, 133 (1967).

[22] V.E. Zakharov, Stability of Periodic Waves of Finite Amplitude on a Surface of Deep Fluid, J. Appl. Mech. Tech. Phys. 2, 190 (1968).

[23] H.C. Yuen and B.M. Lake, Nonlinear Deep Water Waves: Theory and Experiment, Phys. Fluids 18, 956 (1975).

[24] H.C. Yuen and B.M. Lake, Nonlinear Dynamics of Deep-Water Gravity Waves, Adv. Appl. Mech. 22, 67 (1982).

[25] N. Akhmediev, V.M. Eleonskii, and N.E. Kulagin, Generation of a Periodic Sequence of Picosecond Pulses in an Optical Fibre: Exact Solutions, Zh. Eksp. Teor. Fiz. 89, 1542 (1985) [Generation of a Periodic Sequence of Picosecond Pulses in an Optical Fibre: Exact Solutions, Sov. Phys. JETP 61, 894 (1985)].

[26] N. Akhmediev, V.M. Eleonskii, and N. E. Kulagin, Exact First-Order Solutions of the Nonlinear 
Schrödinger Equation, Teor. Mat. Fiz. (USSR) 72, 183 (1987) [Exact First-Order Solutions of the Nonlinear Schrödinger Equation, Theor. Math. Phys. 72, 809 (1987)].

[27] E. A. Kuznetsov, Solitons in a Parametrically Unstable Plasma, Sov. Phys. Dokl. 22, 507 (1977).

[28] Y.C. Ma, The Perturbed Plane Wave Solutions of the Cubic Nonlinear Schrödinger Equation, Stud. Appl. Math. 60, 43 (1979).
[29] N. Akhmediev, A. Ankiewicz, J. M. Soto-Crespo, and J. M. Dudley, Rogue Wave Early Warning Through Spectral Measurements?, Phys. Lett. A 375, 541 (2011).

[30] K. B. Dysthe, Note on the Modification of the Nonlinear Schrödinger Equation for Application to Deep Water Waves, Proc. R. Soc. A 369, 105 (1979).

[31] K. Trulsen, I. Kliakhandler, K. B. Dysthe, and M. G. Velarde, On Weakly Nonlinear Modulation of Waves on Deep Water, Phys. Fluids 12, 2432 (2000). 